\title{
Go4Flex: Goal-oriented Process Modelling
}

\author{
Lars Braubach, Alexander Pokahr, Kai Jander, Winfried Lamersdorf, and \\ Birgit Burmeister
}

\begin{abstract}
Many companies consider business process management strategies a fundamental source for successful business operation. Despite this importance of business processes a conceptual and operational gap still exists between the business and the IT view of processes. In this paper we argue that an important reason for this gap is the strong focus of IT on the behaviour and execution perspective of workflows while more abstract and higher-level process properties are often neglected. This is especially apparent in the way processes are modelled and described on the IT-side using state of the art modelling approaches like BPMN. The presented Go4Flex research project, which is conducted in cooperation with Daimler AG, has the objective of bringing together both sides by establishing higher-level modelling concepts for workflows, which results both in increased intelligibility of workflow descriptions for business people and greater consideration for the way processes are described on the business side. The core idea of the approach is to strengthen the context perspective of a workflow by introducing different kinds of goals and goal relationships in addition to the established activity-centred behaviour model. The applicability of the approach is further illustrated with an example workflow from Daimler AG.
\end{abstract}

\section{Introduction}

Business processes form a challenging research area, in which the business and IT sides have to be conceptually and methodically aligned in order to achieve their full potential [7]. The business side typically focuses on the elicitation of business processes, their management and controlling as well

Lars Braubach - Alexander Pokahr - Kai Jander - Winfried Lamersdorf

Distributed Systems and Information Systems Group, University of Hamburg, Germany, e-mail: \{braubach, pokahr, jander\}@informatik.uni-hamburg.de

Birgit Burmeister

Daimler Group Research, Sindelfingen, Germany, e-mail: birgit.burmeister@ daimler.com 
as their optimization while the IT side has to deal with their simulation, execution as well as real-time monitoring. Currently, the artefacts produced from methods at the business side are only partially adopted by IT so that a conceptual gap still exists. Few approaches have tried to address business process management (BPM) at a holistic level, which is a prerequisite for effective and efficient BPM. One example for such a holistic approach is the ARIS house of business engineering [16], also used at Daimler AG.

In practice, experience has shown that modelling means offered by ARIS, focussing on event process chains (EPCs), are insufficient for some processes at Daimler AG. While processes have been documented with EPCs, they have not been directly adopted by the workflow participants. One major issue is the strong focus on activities and their ordering, found in nearly all modelling languages including BPMN (business process modelling notation) [11]. As the processes considered at Daimler AG can frequently change, the abstractness of the process descriptions is essential for their long-term usefulness.

In this paper, we propose an approach based on the notion of process goals. The use of process goals aims at achieving a higher degree of abstractness in the process models by employing goals to describe what is to be achieved instead of how it should be done. The means for achieving a goal can then be described on a finer grained level using traditional activity oriented workflow languages. In addition, as goals play an important conceptual role on the business side, e.g. in well known process elicitation methods like business score cards [7], policy deployment [7] and with respect to process monitoring and evaluation metrics (key performance indicators, KPIs), they form an ideal basis for enhancing the integration level between both.

The next section will discuss related work with respect to workflow modelling approaches. Thereafter, in Section 3, the goal-oriented modelling approach will be presented in the context of the Go4Flex project. Section 4 further illustrates the approach by explaining selected concepts using an example workflow from Daimler AG and finally, Section 5 concludes the paper and highlights aspects of current and future work.

\section{Related Work}

In the literature many different description languages for workflows can be found. They can be coarsely divided into domain centred modelling approaches and execution oriented approaches. Example of the former group are e.g. EPCs [16], BPMN [11] and YAWL (yet another workflow language) [18], and of the latter group especially BPEL (business process execution language) [12], petri nets [14] and also rule based approaches like ECA (event condition action) [8]. The difference between both kinds of approaches is mainly the level of details supported by the language. Execution languages allow specifying more technical details and can thus be used to describe workflows that can be automatically executed. Yet, there is no fixed border between both, because on the one hand some of the more technical languages also 
offer comprehensible modelling concepts and on the other hand also mechanisms have been devised for converting a modelling language to an executable counterpart, e.g. from BPMN to BPEL [13] or from EPCs to rules [8].

To assess the usefulness of these approaches it is necessary to understand the different perspectives that are relevant during workflow modelling. Based on the early work of Curtis et al. [5], List and Korherr [9] proposed a holistic view on business processes consisting of five perspectives. The functional view focuses on the concrete execution of tasks and typically considers concepts for describing subprocesses and atomic tasks. By using the related behaviour view the sequencing of these elements is controlled. In many cases workflow control patterns like sequence, AND- or XOR splits and joins [17] are used for that purpose. The informational view is related to the process related data elements that are on the one hand necessary for task processing and on the other also produced by tasks. These data elements represent simple information as well as complex objects and business products or services and are often called process resources. In the organizational perspective it is highlighted who processes the tasks and how the distribution of tasks can be handled. Hence, in this view especially concepts like actors, roles and organizational units play an important role. Finally, the context perspective adds an overview or meta perspective to the process, which may contain important process characteristics like the process goals and their performance metrics in form of e.g. key performance indicators (KPIs).

Please note that the first four perspectives are quite commonly agreed upon and also build the fundamental building block of the ARIS house of business engineering [16]. New is the recent addition of the context perspective, which is until now not adequately reflected and connected to the concepts of the other perspectives. Today, most modelling approaches still focus too much on the functional and behaviour perspective, which can e.g. be observed in the BPMN, YAWL and BPEL languages. Organizational aspects are often reduced to the issue of task distribution and for the informational perspective established techniques like the entity relationship model (ERM) are utilized. An exception is the holistic ARIS approach, which pinpoints the right direction but is very heavyweight and offers dozens of modelling diagrams failing to achieve minimality in the sense of the parsimony principle [10] (introduce only as many concepts as really needed). Additionally, at the heart of the approach is the EPC behaviour description, which is a fine-grained task and event based modelling approach without goals.

Missing in all aforementioned approaches is the explicit modelling of the process goals and relating them to the context as well as to the behaviour view, i.e. goals should be used to understand what a process is used for and also directly steer the process execution. In addition the performance metrics, often using KPIs, form another important brick of the context perspective that currently gains much practical attention through the advent of real-time business activity monitoring (BAM) tools. Therefore, several BPMN tools try to offer solutions for the KPI modelling and process linking but fail altogether 
to link the KPI results to the underlying process goals, simply because they are not represented. Similar to our research objective is the approach of [15], who use the user requirements notation (URN) together with use case maps (UCM) and the goal-oriented requirements language (GRL) for modelling the context perspective. Also the idea of goal, KPI and process linking has been pushed forward in their approach. One main difference is that we use goals for functional and non-functional process aspects and hence make goals an integral conceptual element for the context and the behaviour perspective. i.e. the goal modelling is considered as starting point for the process definitions according to the goal-context method of developed by Daimler AG [3], which has also been implemented in a commercial tool [4].

\section{Go4Flex Approach}

The Go4Flex project aims at providing advanced conceptual and software technical means for modelling and executing complex business processes. Go4Flex advocates a unifying approach that integrates the five aforementioned dimensions of workflows into a consistent framework. This framework is based on concepts, which have been developed in the area of agents and multi-agent systems. Existing multi-agent systems concepts cover large parts of the perspectives relevant for workflows and additionally are based on the unifying metaphor of (software) agents. The main research question of the DFG-funded Go4Flex project is to isolate interesting multi-agent ideas and make them usable also for workflows. Most importantly, Go4Flex focuses on the behaviour and context perspectives, which suffer among other things from their low conceptual connectivity. Thus Go4Flex is based on the notion of declarative goals $[2,19]$. On the one hand goals allow capturing the reason for executing process activities. This facilitates taking a top-down perspective on processes that starts from high-level business goals instead of having to focus on low-level activities. On the other hand goals are an ideal concept for understanding the process context (why something is done and how good it is done). Thus we envision goals as one fundamental conceptual entity for both perspectives. Besides goals, the approach is conceived to integrate well with established concepts, e.g. by reusing available BPM concepts and techniques.

\subsection{Concepts}

Regarding the behaviour perspective, the Go4Flex approach is based on the goal-context method developed by Daimler Group Research [3]. This method advocates to model processes by starting from high-level goals. The process is refined by decomposing goals into a goal hierarchy. For each goal, the designer can specify a number of declarative properties, e.g. normal conditions to be fulfilled for a goal to be regarded as achieved or exceptional conditions, denoting when a goal should not be pursued. Once the goal hierarchy reaches 
an appropriate level of detail, the designer can start modelling plans to fulfil the leaf goals of the goal hierarchy. Plans represent courses of action that capture one of potentially many alternatives for fulfilling a superordinated goal. During process execution, the workflow engine automatically selects appropriate plans for each goal based on conditions that state the applicability of each plan to a given situation. Moreover, if a plan fails, the engine can try out other plans to achieve the same goal, if alternative plans are available.

Therefore, the method proposes two abstraction levels for process modelling: At the goal level, the process is modelled in a declarative way as a tree structure of goals to be fulfilled for the process to be successful. At the plan level, concrete activities for handling specific goals are described. For the goal level, a new notation is introduced. The plan level uses the existing BPMN as modelling language. The goal hierarchy represents the declarative properties of the process (conditions to be fulfilled), while the plans capture procedural aspects (sequences of actions to be executed).

The representation and execution semantics for the goal level has been directly adapted from the notion of goals in mentalistic belief-desire-intention (BDI) agents [2][1]. In order to simplify the goal semantics different functional goal kinds have been proposed, from which the most important ones are achieve, maintain, query and perform goals. These goal kinds map to different application use cases and thus help in naturally modelling the problem at hand. Achievement goals are the most common goal kind, which aim at the establishment of a user defined world state (declaratively expressed as a target condition). The goal executes plans until its condition is met or no more plans are available. On the other hand, a maintenance goal can be used to ensure that a specific world state is preserved. In case of a violation the goal tries to re-establish the condition by executing as many plans as needed. A query goal is used for information retrieval and only executes plans when the requested piece of information is not already available in the process context. Finally, perform goals are the simplest form of goals, which have a procedural semantics and are directly connected to executing possibly several plans. A detailed description of these goal kinds can be found in [2].

With respect to the context perspective our approach is inspired by the ideas of [15], who propose to use non-functional (soft) goals for expressing process performance metrics. In contrast to their exclusive treatment of nonfunctional goals, we envision that they should be part of the functional goal hierarchy similar as in the agent methodology Tropos [6]. The connection between both is described with contribution links, which means that a functional or non-functional element either positively or negatively contributes to the non-functional goal. On lower levels non-functional goals can be decomposed into KPIs, which exactly determine the metrics of the non-functional goal and how it is measured. The explicit relationships between functional and non-functional process elements facilitate the way measurement results are interpreted, because their context is apparent. 


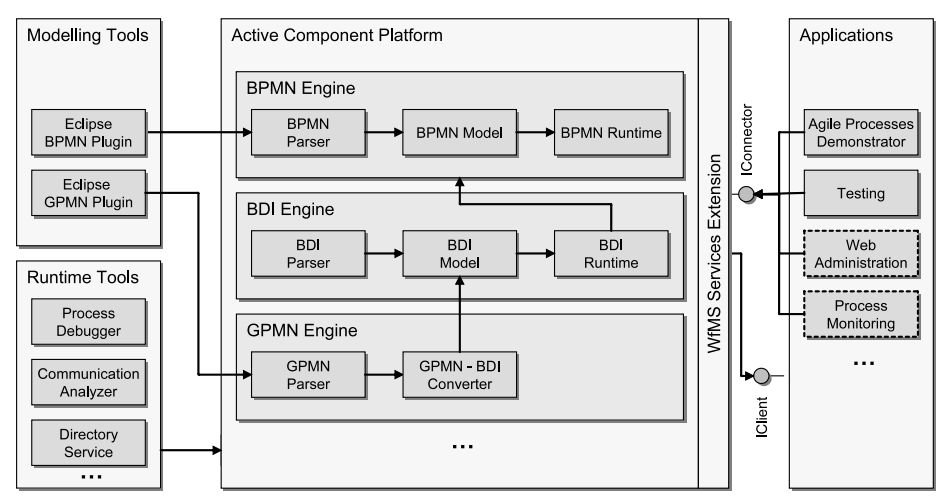

Fig. 1 Go4Flex architecture

Taken together the described approaches have several advantages compared to traditional techniques. First, the understandability of workflows is fostered by introducing goals as conceptual entities abstracting away from purely procedural descriptions (unlike e.g. BPMN). Second, the process description naturally facilitates design and runtime agility. Runtime agility is achieved by executing different plans for the same goal, depending on the current situation. Design agility results from that fact that the process descriptions are open to future changes and extensions. E.g. a process designer can add another plan for an alternative course of action without changing any of the existing process descriptions. Such an incremental process development is a very natural approach, because business users often prefer thinking in scenarios and not in complete processes, that include every potential alternative. Third, the conceptual integration of the context with the behaviour perspective will allow meaningful business process monitoring, because the metrics and KPIs are directly related to process goals.

\subsection{Implementation}

A number of necessary tools for modelling and execution of goal-based processes have been developed by the Go4Flex project. Figure 1 shows four key areas for tool support (modelling tools, runtime tools, platform, and applications). The starting point for creating new workflows are the modelling tools. Two editors are provided for developing new workflow models (see Figure 2, left). A graphical editor provides a special notation called GPMN (Goaloriented Process Modelling Notation) which allow users to create Go4Flex goal hierarchies. This GPMN editor is also used to define the context of the workflow. The BPMN editor is used to create BPMN workflow fragments (i.e. plans), but can also be used to develop standalone BPMN workflows.

The workflow models generated by the editors can be used to instantiate workflows using the Jadex Active Component Platform ${ }^{1}$. The platform

\footnotetext{
1 http://jadex.sourceforge.net
} 


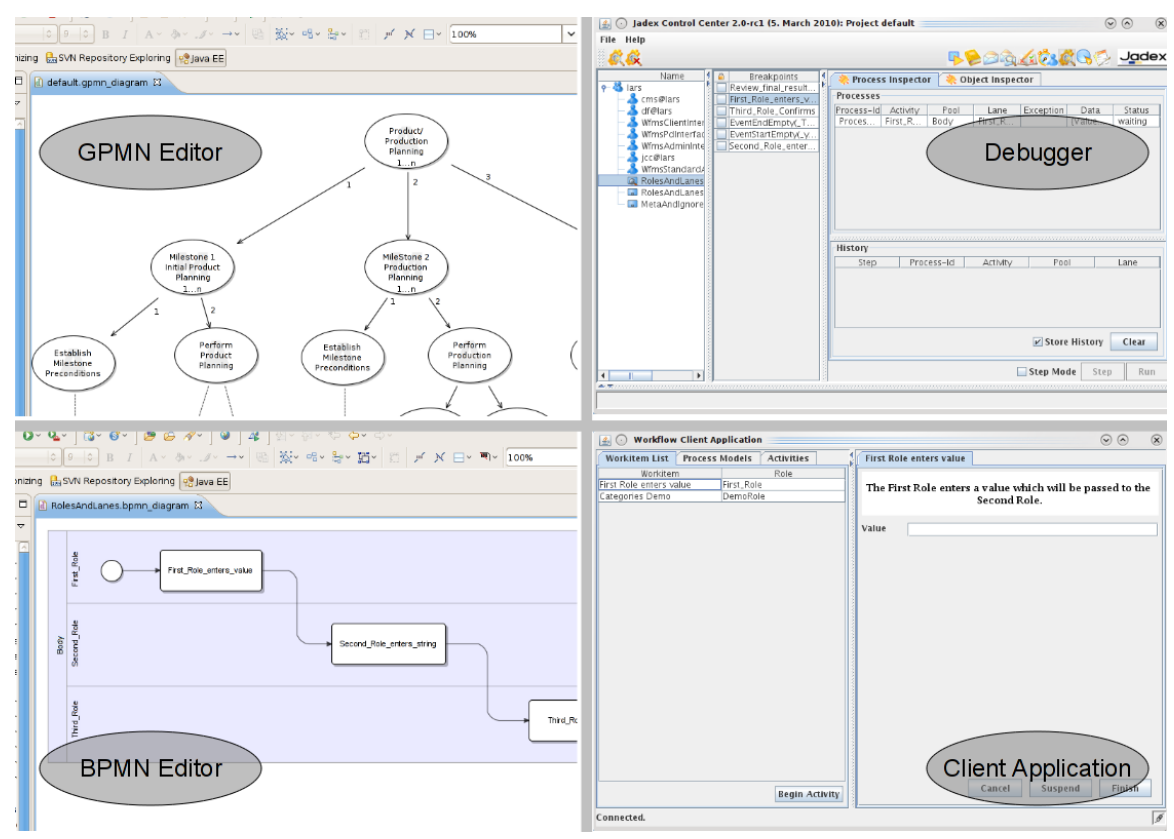

Fig. 2 Tools implemented for use in Go4Flex.

includes an interpreter for BPMN workflows or workflow fragments. GPMN workflows are supported by using a converter, which translates the GPMN model into a BDI agent model that can be executed by the BDI runtime.

Runtime tools (see Figure 2, right) provide diagnostics for running workflows. This includes a process debugger, allowing introspection, break points and stepped execution of workflows, a communication analyzer enabling the user to monitor and record messages passed between workflows and a directory service for deploying new workflow models at runtime.

User applications interact with the system from the perspective of a workflow participant. Currently, a GUI-based workflow client application and an automated workflow testing tool are available. Future additions could include a web-based administration tool and process monitoring tools. User applications interact with the runtime environment through a workflow management system services extension of the active component platform, adding additional runtime features like role management and work item handling.

\section{Example Workflow}

The Go4Flex goal concept has been used in several different areas at Daimler AG. The simplified digital production planning workflow shown in Figure 3 is an example how the new approach can be used in practice. The root of the goal hierarchy is an achieve goal which represents the general objective of the 


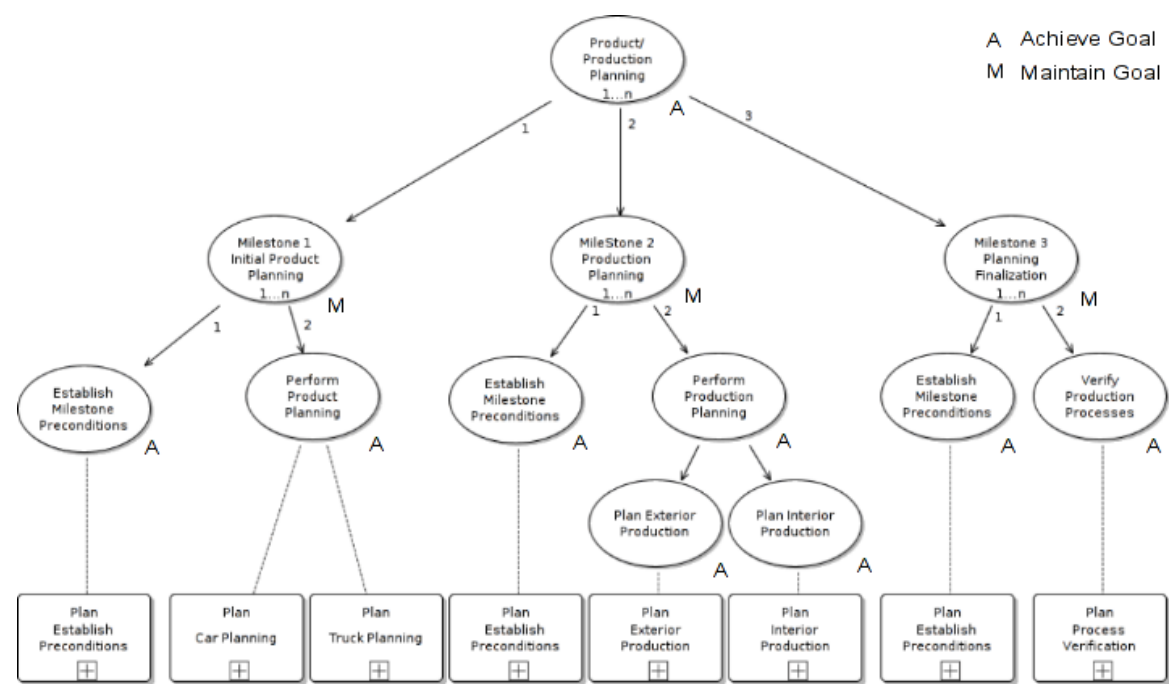

Fig. 3 Goal hierarchy structure as used in the digital production workflow

workflow. ${ }^{2}$ The second layer of subgoals represents process milestones. The first milestone is the product planning stage, followed by planning the production processes of the product as the second milestone. The third milestone verifies the processes and finalizes the planning. The top goal is sequential, the next milestone goal only starts once the previous milestone has finished.

Employing milestones as the second layer of the workflow allows quick assessment of workflow progress by following the active milestone goal. For example, if the production planning milestone goal is active, the product planning stage has already finished. As alternative the second layer of goals can be structured by the area of operation and the milestone steps are delegated as subgoals further down the hierarchy. While this would be a reasonable design, it lacks the monitoring advantage of the former approach.

Instead, milestone subgoals have their subgoals tied to areas of operation. If possible, they can be executed in parallel. For example, planning the fittings and equipment of the interior can be done independently of exterior features like colour. If this is not viable, the goal can be declared sequential.

Once the hierarchy approaches a sufficiently fine-grained level, plans can be added to the goals. Plans represent BPMN workflow fragments which describe actions necessary to reach the goal. Since the goal hierarchy already decomposed the complex workflow goal into more basic goals, BPMN plans can be relatively simple, like requesting and storing information.

Milestone goals are modelled as maintain goals, monitoring whether the state of the context diverges from what the milestone is supposed to establish. In this case, the product planning goal monitors whether the conditions for a planned product are met. Once the top goal activates it, the context implies

2 The original workflow has been made abstract due to business secrecy reasons. 
that product planning is necessary, activating the subgoals in the subtree. When finished, the top level goal activates the production phase goal.

However, the previous milestone goal is still actively monitoring the context. If the production planning invalidates assumptions made during the product planning, the product planning goal reactivates. Context conditions pause later milestone goals while the product planning milestone goal attempts to re-establish a product plan. Even though the whole subtree reactivates, subgoals that are leaf goals (i.e. goals without further subgoals) contain target conditions specifying their required context state. If the condition is already met, the goal finishes without plan execution. As a result, only unfulfilled subgoals will execute their respective plans a second time.

This illustrates how the Go4Flex approach allows flexible workflows. If a planning error invalidates work done in a previous milestone, the milestone reactivates and solves the problem without restarting the workflow. Thus the workflow reacts to changes in the context and maintains a consistent state.

\section{Conclusion}

In this paper it has been argued that a conceptual gap between the business and IT side of business processes still exists due to the many perspectives that have to be considered. In the IT area there is still a strong tendency to overweigh the importance of the behaviour perspective, which is clearly reflected in the state of the art modelling approaches like BPMN. In contrast, the context perspective, which represents a business near meta view on the reasons for process execution and its metrics, has not been subject of extensive technical research and is thus poorly supported from IT side.

The Go4Flex project targets this research strand by exploiting concepts from multi-agent systems for improving the conceptual underpinnings especially in the context perspective and its relation to the behaviour view. One fundamental building block of Go4Flex is the usage of declarative goals, which increase the understandability and abstractness of workflow descriptions. On the one hand functional goals help representing the underlying reasons for executing a process (enhancement of the behaviour view) and on the other hand non-functional goals support the understanding of the process metrics (enhancement of the relation between context and behaviour view).

In order to explore the new concepts workflow modelling, execution and management systems have been developed. The execution engine is currently capable of executing goal-oriented (GPMN) as well as BPMN workflows. The system is based on the Jadex active components platform, which is a generic infrastructure for running heterogeneous active elements and provides a suite of runtime tools for their management like debugging and conversation analysis. The engine for GPMN processes reuses an available BDI agent engine by converting the goal model to a BDI agent representation. To show the practical usefulness of the approach (an abstract version of) an example workflow from our project partner Daimler has been presented. 
Future work will further develop and systematize the available modelling concepts. One aspect in this direction is the description of often recurring modelling fragments in form of goal-oriented workflow patterns. Furthermore the integration of non-functional goals represents an important step in the direction of goal-oriented process monitoring and improvement.

Acknowledgements We would like to thank the DFG for supporting the technology transfer project Go4Flex.

\section{References}

1. L. Braubach and A. Pokahr. Representing long-term and interest bdi goals. In Proc. of (ProMAS-7), pages 29-43. IFAAMAS Foundation, 52009.

2. L. Braubach, A. Pokahr, D. Moldt, and W. Lamersdorf. Goal Representation for BDI Agent Systems. In Proc. of (ProMAS 2004), pages 44-65. Springer, 2005.

3. B. Burmeister, M. Arnold, F. Copaciu, and G. Rimassa. Bdi-agents for agile goal-oriented business processes. In $A A M A S$ '08, pages 37-44. IFAAMAS, 2008.

4. M. Calisti and D. Greenwood. Goal-oriented autonomic process modeling and execution for next generation networks. In Proc. of MACE '08. Springer, 2008.

5. B. Curtis, M. Kellner, and J. Over. Process modeling. Commun. ACM, 35(9):7590, 1992.

6. P. Giorgini, M. Kolp, J. Mylopoulos, and M. Pistore. The Tropos Methodology. In Methodol. and Software Eng. for Agent Systems, pages 89-106. Kluwer, 2004.

7. W. Sesselmann H. J. Schmelzer. Geschäftsprozessmanagement in der Praxis. Hanser Fachbuchverlag, 2008.

8. G. Knolmayer, R. Endl, and M. Pfahrer. Modeling processes and workflows by business rules. In Business Process Management, Models, Techniques, and Empirical Studies, pages 16-29, London, UK, 2000. Springer-Verlag.

9. B. List and B. Korherr. An evaluation of conceptual business process modelling languages. In Proc. of SAC '06, pages 1532-1539. ACM, 2006.

10. A. Newell. Unified Theories of Cognition. Harvard University Press, 1990.

11. Object Management Group (OMG). Business Process Modeling Notation $(B P M N)$ Specification, version 1.1 edition, February 2008.

12. Organization for the Advancement of Structured Information Standards (OASIS). Web Services Business Process Execution Language (WSPBEL) Specification, version 2.0 edition, 2007.

13. C. Ouyang, M. Dumas, A. ter Hofstede, and W. van der Aalst. From bpmn process models to bpel web services. In Proc. of ICWS '06, pages 285-292. IEEE, 2006.

14. C.A. Petri. Kommunikation mit Automaten. PhD thesis, Institut fur Instrumentelle Mathematik, Bonn, 1962.

15. A. Pourshahid, D. Amyot, L. Peyton, S. Ghanavati, P. Chen, M. Weiss, and A. Forster. Business process management with the user requirements notation. Electronic Commerce Research, 9(4):269-316, 2009.

16. A.-W. Scheer and M. Nüttgens. Aris architecture and reference models for business process management. In Business Process Management. Springer, 2000.

17. W. M. P. van Der Aalst, A. H. M. Ter Hofstede, B. Kiepuszewski, and A. P. Barros. Workflow patterns. Distrib. Parallel Databases, 14(1):5-51, 2003.

18. W. M. P. van der Aalst and A. H. M. ter Hofstede. Yawl: yet another workflow language. Information Systems, 30(4):245-275, June 2005.

19. M. Winikoff, L. Padgham, J. Harland, and J. Thangarajah. Declarative \& Procedural Goals in Intelligent Agent Systems. In Proc. of KR 2002. Morgan Kaufmann Publishers, 2002. 